\title{
Avoiding Pneumocephalus after Chronic Subdural Hematoma Evacuation: The Temporary Double Drainage Technique
}

\section{Cecchini G*}

Department of Neurosurgery, Universita Politecnica delle Marche, Italy

\begin{abstract}
Aim: Chronic subdural hematoma presents an increasing challenge as the population ages. Despite the plethora of surgical techniques described, a high recurrence rate is still encountered. One of the possible risk factor for recurrence is post-operative pneumocephalus. The technique described is focused on avoiding post-operative pneumocephalus and obtaining complete hematoma evacuation.
\end{abstract}

Methods: We describe a simple surgical technique consisting of a temporary double air-fluid inverse drainage.

Results: The technique proposed in this paper allows the complete evacuation of the hematoma fluid and, at the same time, the removal of intracranial air employing the same devices normally used in the standard procedure.

Conclusion: The technique proposed in this paper is safe, simple and could be highly effective.

Keywords: Chronic subdural hematoma; Pneumocephalus; Recurrence; Double drainage technique

\section{Introduction}

Chronic subdural hematoma (CSDH) is an increasingly common neurosurgical disease $[1,2]$. Its pathophysiology involves inflammation, impaired hemostasis, angiogenesis [3,4]. These factors are mainly mediated by cytokines harbored in the hematoma fluid $[5,6]$. Thus, the main aim of surgery should be the complete removal of this liquid $[7,8]$. Despite the numerous techniques described to evacuate CSDH, the recurrence rate after burr hole surgery ranges from 9.2 to $26.5 \%$ [9]. Previous studies identified several risk factors for recurrence including advanced age, bleeding tendency, brain atrophy, alcohol abuse, bilateral CSDH, hematoma density, diabetes mellitus, arachnoid cyst, postoperative posture, inflammation cytokines, and some technical aspects of surgery $[9,10-14]$. Among them, postoperative subdural air accumulation has been advocated $[9,12,15,16]$.

The aim of the technique presented in this paper, is to reduce or eliminate the postoperative accumulation of air in the subdural space and accomplish the complete evacuation of the inflammatory fluid.

\section{Materials and Methods}

One of the major advantages of this technique is that it is tailored for surgeons approaching CSDH under local anesthesia: in the awake patient, it could be difficult to place the burr hole trephination at the highest point (to reduce pneumocephalus) and over the thickest part of the hematoma at once. In fact, these two objective can be targeted at the same time only with general anesthesia because of the need of the lateral decubitus. Many surgeons prefer local anesthesia to reduce surgical morbidity because of the avoidance of endotracheal intubation. Under local anesthesia, the surgical approach is usually performed in the supine position with the head slightly turned toward the opposite side of the hematoma. The head of the patient may be fixed with adhesive tape passing over the forehead to the head of bed, which is generally elevated to $15-20^{\circ}$.

The hematoma is approached over the thickest part, usually on the parietal boss (Figure 1). The skin incision is curvilinear (thus reducing the possibility of impaired wound healing due to the presence of the burr hole directly under the incision) and is brought into alignment with an eventual future skin flap for craniotomy.
The burr hole is not circular, but slightly elongated, oriented along with the longitudinal axis of the head (Figure 1). It is carried out with a pneumatic drill, possibly avoiding Kerrison rongeur, to reduce epidural bleeding. Hemostasis is achieved applying bone wax. The elliptical shape of the craniectomy is one of the keypoints of this technique.

The dura is gently coagulated and opened in two different points representing the focus points of the ellipsoid craniectomy (Figure 2). Both the openings are performed in a stellate fashion. The two openings allow the generation of an open system that can be switched to a closed one simply inserting two catheters. The posterior opening should be slightly larger than the anterior one, allowing the future entrance of a Jackson Pratt (JP) drain (Figure 2). We take this chance to remind the

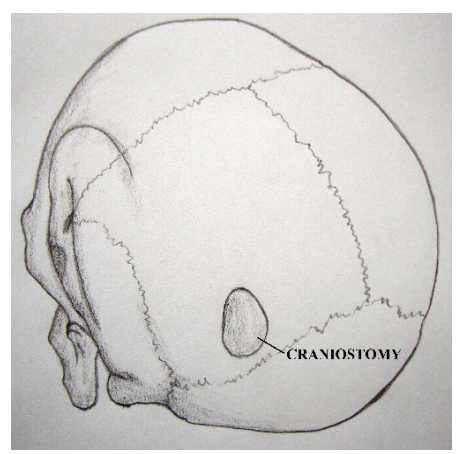

Figure 1: The drawing shows the position of the elliptical craniectomy, usually placed posterior to the parietal eminence. The placement of the burr hole should be over the thickest part of the hematoma.

*Corresponding author: Giulio Cecchini, Department of Neurosurgery Universita Politecnica delle Marche, Italy, Tel: 004407492550206; E-mail: cecchinigiulio@hotmail.it

Received August 20, 2015; Accepted August 27, 2015; Published August 31, 2015

Citation: Cecchini G (2015) Avoiding Pneumocephalus after Chronic Subdural Hematoma Evacuation: The Temporary Double Drainage Technique. J Neurol Disord S1: 001. doi:10.4172/2329-6895.S1-001

Copyright: (c) 2015 Cecchini G. This is an open-access article distributed under the terms of the Creative Commons Attribution License, which permits unrestricted use, distribution, and reproduction in any medium, provided the original author and source are credited. 


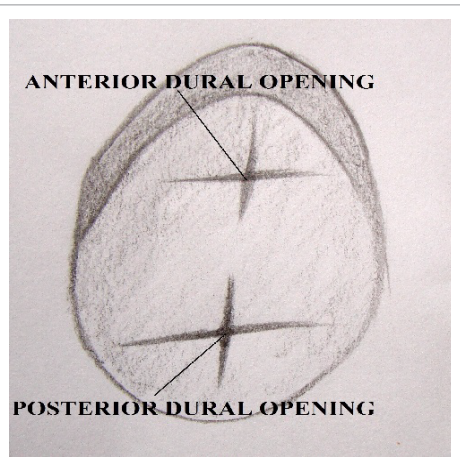

Figure 2: In the drawing the elliptical craniectomy is represented. The underlying dura has been cut in a stellate fashion. The anterior opening will give access to a Foley catheter, the posterior one will initially allow the hematoma to egress and finally the JP drain to be placed in the subdural space. The posterior opening is larger than the anterior.

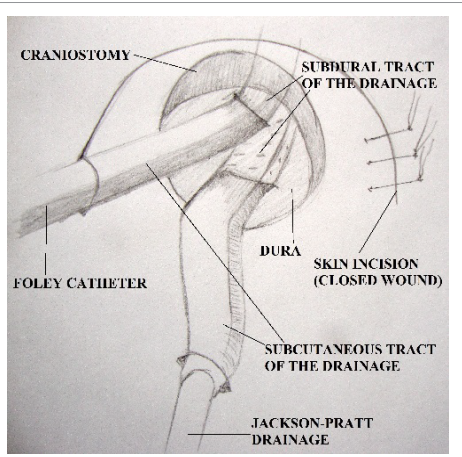

Figure 3: See-through drawing representing the "C" shaped skin incision (the galea has already been closed, skin suture have been partially applied) with both catheter entering into the subdural space. The JP drain is tunneled through the galea, the Foley catheter goes through the tip of the skin incision.

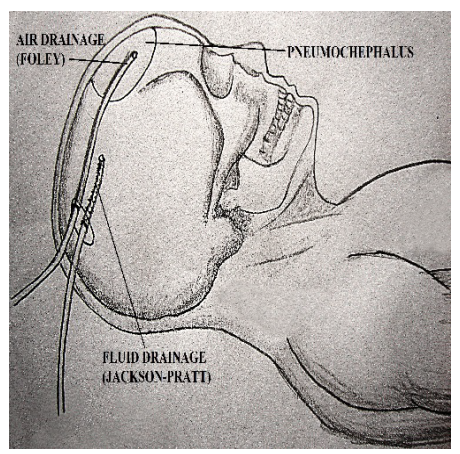

Figure 4: Drawing showing the location of intracranial air in the supine position and the ideal position of the catheters.

reader the importance of opening the dura preventing a sudden drop of intracranial pressure [17].

A Foley or a Nelaton catheter is inserted in the anterior opening. A $50 \mathrm{~mL}$ syringe in connected to the catheter and irrigation is continued until complete removal of the inflammatory fluid via the inferior opening.

The Foley catheter is then taken out of the subdural space, washed, and put back into the anterior opening until it reaches about the frontal pole (or the highest point of the subdural space, considering the position of the head). It is connected to a $20 \mathrm{~mL}$ syringe.

A JP drain, after being tunneled through the galea, is inserted in the subdural space via the posterior dural opening, pointing the frontal lobe (Figure 3).

The galea is closed with 3-0 inverted Vicryl stitches in a watertight fashion, giving only access to the Foley catheter (Figure 3).

Normal saline is infused through the JP catheter while air is gently aspirated via the Foley catheter (Figure 4). This phase continues until no more air comes back from the subdural space. The Foley catheter is then removed and skin is closed.

The JP drain closed system is left in situ for 48 hours. Then, when removing the JP catheter, it is crucial to prevent any air passage through its multiple holes applying a gentle pressure over the skin, close to the exit point.

\section{Discussion}

The main technical nuances that distinguish this technique from the previous ones are the following:

1) The elliptical craniectomy.

2) The dural opening in two different points (the posterior larger than the anterior).

3) The employment of two drains (a Foley catheter for gentle air aspiration, a JP for normal saline infusion).

4) The possibility to switch from an open system to a closed system and vice versa easily and quickly.

The rationale of this technique, in fact, is the use of the benefits of the two main surgical technique: the "closed" technique doesn't permit any air entrance, but the hematoma fluid is not replaced by normal saline rinse. The "open" technique allows complete washing of the hematoma inflammatory fluid, but postoperative pneumocephalus is the expected side-effect [18].

In a recent letter to the editor, Gelabert-Gonzàlez et al. remark upon a previous paper $[18,19]$. Some key elements are pointed out:

1) The preference for local anesthesia. General anesthesia used on elderly with multiple concomitant diseases may not be the best option. The main advantage of general anesthesia is the possibility to place the patient in the side or prone position in order to turn the burr hole to the top of the calvaria, thus reducing post operative pneumocephalus. Of course, patient compliance might decrease in the side or prone position when surgery is performed under local anesthesia. The technique we suggest in this paper is performed in the supine position but it offers, at the same time, an easy solution to avoid pneumocephalus.

2) The importance of irrigation. Although irrigation applied to an open system may lead to pneumocephalus, washing out all the hematoma fluid is the objective of the procedure [7,20,21]. The temporary double drain technique may prevent pneumocephalus and allows performing irrigation with inflammatory fluid removal.

The procedure described consists therefore of a temporary double inverse air-fluid drainage.

The technique proposed in this paper is safe, simple and could be highly effective. Clinical trials are required to clarify its efficacy.

\section{References}

1. Abouzari M, Rashidi A, Rezaii J, Esfandiari K, Asadollahi M, et al. (2007) The role of postoperative patient posture in the recurrence of traumatic chronic subdural hematoma after burr-hole surgery. Neurosurgery 61: 794-797. 
Citation: Cecchini G (2015) Avoiding Pneumocephalus after Chronic Subdural Hematoma Evacuation: The Temporary Double Drainage Technique. J Neurol Disord S1: 001. doi:10.4172/2329-6895.S1-001

2. Cecchini G (2015) Chronic subdural hematoma pathophysiology: a unifying theory for a dynamic process. J Neurosurg Sci.

3. Dinc C, Iplikcioglu AC, Bikmaz K, Navruz Y (2008) Intracerebral haemorrhage occurring at remote site following evacuation of chronic subdural haematoma. Acta Neurochir (Wien) 150: 497-499.

4. Dumont TM, Rughani Al, Goeckes T, Tranmer BI (2013) Chronic subdural hematoma: a sentinel health event. World Neurosurg 80: 889-892.

5. Emich S, Dollenz M, Winkler PA (2015) Burr hole is not burr hole: technical considerations to the evacuation of chronic subdural hematomas. Acta Neurochir (Wien) 157: 497-499.

6. Gelabert-González M, Rico-Cotelo M, Arán-Echabe E (2015) Burr hole is not burr hole. Acta Neurochir (Wien).

7. Hennig R, Kloster R (1999) Burr hole evacuation of chronic subdural haematomas followed by continuous inflow and outflow irrigation. Acta Neurochir (Wien) 141: 171-176.

8. Hong H-J, Kim Y-J, Yi H-J, Ko Y, Oh S-J, et al. (2009) Role of angiogenic growth factors and inflammatory cytokine on recurrence of chronic subdural hematoma. Surg Neurol 71: 161-165.

9. Ihab Z (2012) Pneumocephalus after surgical evacuation of chronic subdura hematoma: Is it a serious complication? Asian J Neurosurg 7: 66-74.

10. Kalamatianos T, Stavrinou LC, Koutsarnakis C, Psachoulia C, Sakas DE, et al. (2012) PIGF and sVEGFR-1 in chronic subdural hematoma: implications for hematoma development. J Neurosurg 118: 1-5.

11. Kuroki T, Katsume M, Harada N, Yamazaki T, Aoki K, et al. (2001) Strict closedsystem drainage for treating chronic subdural haematoma. Acta Neurochir (Wien) 143: 1041-1044

12. Lindvall P, Koskinen $L$ (2009) Anticoagulants and antiplatelet agents and the risk of development and recurrence of chronic subdural haematomas. $\mathrm{J}$ Clin Neurosci.

13. Liu W, Bakker NA, Groen RJM (2014) Chronic subdural hematoma: a systematic review and meta-analysis of surgical procedures. J Neurosurg 121: 665-73.

14. Miranda LB, Braxton E, Hobbs J, Quigley MR (2011) Chronic subdural hematoma in the elderly: not a benign disease. J Neurosurg 114: 72-76.

15. Nakaguchi H, Tanishima T, Yoshimasu N (2001) Factors in the natural history of chronic subdural hematomas that influence their postoperative recurrence. J Neurosurg 95: 256-262.

16. Osuka K, Watanabe Y, Usuda N, Atsuzawa K, Shima H, et al. (2013) Activation of JAK-STAT3 signaling pathway in chronic subdural hematoma outer membranes. Neurosci Lett 534: 166-170.

17. Santarius T, Kirkpatrick PJ, Ganesan D (2009) Use of drains versus no drains after burr-hole evacuation of chronic subdural haematoma: a randomised controlled trial. Lancet 374: 1067-1073.

18. Stanišić M, Hald J, Rasmussen IA, Pripp AH, Ivanović J, et al. (2013) Volume and densities of chronic subdural haematoma obtained from CT imaging as predictors of postoperative recurrence: a prospective study of 107 operated patients. Acta Neurochir (Wien) 155: 323-333.

19. Torihashi K, Sadamasa N, Yoshida K, Narumi O, Chin M, Yamagata S (2008) Independent predictors for recurrence of chronic subdural hematoma: a review of 343 consecutive surgical cases. Neurosurgery 63: 1125-1129.

20. Weigel R, Schmiedek P, Krauss JK (2003) Outcome of contemporary surgery for chronic subdural haematoma: evidence based review. J Neurol Neurosurg Psychiatry 74: 937-943.

21. Wilberger JE (2000) Pathophysiology of evolution and recurrence of chronic subdural hematoma. Neurosurg Clin N Am 11: 435-438.
This article was originally published in a special issue, Management of Neurosurgical Complications handled by Editor(s). Dr. Athanasios K. Petridis, Department of Neurosurgery, University of Schleswig Holstein, Germany 